\title{
Atomic force microscopy of confined liquids using the thermal bending fluctuations of the cantilever
}

\author{
Fei Liu, ${ }^{*}$ Sissi de Beer, ${ }^{\dagger}$ Dirk van den Ende, and Frieder Mugele \\ Physics of Complex Fluids, MESA + Institute for Nanotechnology, University of Twente P. O. Box 217, 7500 AE Enschede, The Netherlands
}

(Received 16 April 2013; published 21 June 2013)

\begin{abstract}
We use atomic force microscopy to measure the distance-dependent solvation forces and the dissipation across liquid films of octamethylcyclotetrasiloxane (OMCTS) confined between a silicon tip and a highly oriented pyrolytic graphite substrate without active excitation of the cantilever. By analyzing the thermal bending fluctuations, we minimize possible nonlinearities of the tip-substrate interaction due to finite excitation amplitudes because these fluctuations are smaller than the typical $1 \AA$, which is much smaller than the characteristic interaction length. Moreover, we avoid the need to determine the phase lag between cantilever excitation and response, which suffers from complications due to hydrodynamic coupling between cantilever and fluid. Consistent results, and especially high-quality dissipation data, are obtained by analyzing the power spectrum and the time autocorrelation of the force fluctuations. We validate our approach by determining the bulk viscosity of OMCTS using tips with a radius of approximately $1 \mu \mathrm{m}$ at tip-substrate separations $>5 \mathrm{~nm}$. For sharp tips we consistently find an exponentially decaying oscillatory tip-substrate interaction stiffness as well as a clearly nonmonotonic variation of the dissipation for tip-substrate distances up to 8 and $6 \mathrm{~nm}$, respectively. Both observations are in line with the results of recent simulations which relate them to distance-dependent transitions of the molecular structure in the liquid.
\end{abstract}

DOI: 10.1103/PhysRevE.87.062406

PACS number(s): 68.08.-p, 66.20.-d, 07.79.Lh, 62.10.+s

\section{INTRODUCTION}

Understanding the properties of nano-confined liquids is of great importance in numerous research fields, like biophysics [1] and nanofluidics [2], and industrial applications, such as friction, wear, and lubrication [3,4]. In particular, what happens when we squeeze out a liquid between atomically flat surfaces? At distances significantly larger than the molecule size, the drainage process is described by the well-known Reynolds approximation [5] of the Navier-Stokes equations. However, for nano-confined liquids, where the film thickness is comparable to the molecular size, continuum physics breaks down and the liquid film is squeezed out layer by layer [6,7]. These discrete transitions and the layering configuration are caused by the molecular self-assembly of the liquid close to solid walls. On confinement this gives rise to the conservative oscillatory solvation forces [8]. These solvation forces were first measured in the 1980s [9] and are by now well established [10-27]. However, how molecular self-assembly affects the dynamics of the confined liquid is still heavily debated due to contradicting experimental results.

In surface forces apparatus (SFA) experiments, confinement-induced solidification was observed when shearing the confined liquid [28,29]. Other studies [13] reported a viscoelastic shear response akin to jamming. Measurements of the rupture process of squeezing out the liquid layer by layer could be described using a discretized version of the Navier-Stokes equations with a more or less bulklike viscosity down to the last two layers [14,20]. Recent experiments [22] and theoretical studies [30] indicate that some of these apparent inconsistencies can be traced down to

\footnotetext{
*f.liu-1@utwente.nl

${ }^{\dagger}$ Present address: Jülich Supercomputing Centre, Wilhelm-JohnenStrasse, 52425 Jülich, Germany.
}

the strong structural anisotropy in the confined liquid, which can lead to a highly anisotropic effective viscosity.

In more recent atomic force microscopy (AFM) experiments with confined liquids similar discrepancies have been observed. While some studies report a monotonic increase in the viscous dissipation [10,19,26,27], others detect distancedependent features in the dissipation [11,12,17,18,21,2325,31,32]. All these measurements were performed using various forms of dynamic AFM spectroscopy using actively driven cantilevers. Several problems may contribute to the discrepancies of the results reported in the literature. In liquid the cantilever is subject to a strong mechanical coupling with the fluid. As a consequence, the cantilever suffers from viscous friction with the ambient fluid leading to a low overall quality factor of order unity. The damping due to the confined liquid is only a rather small addition to the overall damping. Moreover, the coupling among the cantilever, the fluid, and the surrounding liquid cell can give rise to additional resonances, leading to the well-known problem of a "forest of peaks", in particular for acoustically driven cantilevers [26,33].

Optimizations of the cantilever holder [34,35], dynamic models taking into account the base motion of the cantilever [26,36], as well as other (e.g., magnetic) driving schemes [37-39] help to reduce these problems, yet they do not overcome the fundamental problem that the reconstruction of the force is based on an inversion of the measured amplitude and phase (or resonance frequency) and requires an accurate model of the cantilever dynamics, including, in particular, knowledge and calibration of the phase lag between driving and response [40,41]. In addition, the finite drive amplitude (e.g., in excess of the molecular diameter) may "smear out" variations of interaction and dissipation forces on smaller length scales [24].

To avoid these difficulties, we revisit the analysis of the thermal noise signal to study the conservative and dissipative properties of confined liquids [15,42-47]. This method, which 
has by now become a standard tool to determine the cantilever spring constant [42], minimizes the external perturbation of the system and it eliminates the need of a phase measurement. We investigate the influence of the tip-substrate interaction on the thermal noise signal to determine both the distance-dependent interaction stiffness due to conservative (oscillatory) tipsubstrate interactions $[15,43,44]$ and the interaction damping due to the local energy dissipation near the substrate. Our approach extends earlier studies of viscoelastic properties of polymeric systems [45-47] and shear forces in confined liquids [48]. We record time series of the noise signal using high-speed and broadband data capture and analyze both their power spectral density (PSD) as well as the time autocorrelation function (ACF) [49]. Notwithstanding earlier reports of differences regarding the effect of electronic noise [50], we find that both approaches yield consistent results, as expected based on the Wiener-Khinchin theorem $[51,52]$.

The conservative force and dissipation in the confined liquid are determined alternatively by fitting the obtained power spectra and autocorrelation of the fluctuating tip displacement to a simple harmonic oscillator (SHO) model of the cantilever. The amplitude of the cantilever motion, typically $50 \mathrm{pm}$ at room temperature, is significantly smaller than that in dynamic AFM, thereby minimizing sample perturbation and ensuring the applicability of linear response. To validate the thermal noise method we first determine the bulk viscosity of the liquid by measuring the hydrodynamic dissipation using relatively large cantilever tips, with a radius of about $1 \mu \mathrm{m}$. This verification is referred to as the Reynolds damping measurement in the paper. Next, we study the distance-dependent interaction forces and dissipation, using smaller tips with a radius of about $50 \mathrm{~nm}$. Both the observed stiffness and damping oscillate as a function of the tip-substrate distance at distances below $6 \mathrm{~nm}$ as a consequence of the layering effects, in agreement with statistical physics [53] and molecular dynamics (MD) simulations [54].

\section{METHODS}

\section{A. Materials}

The experimental AFM setup to obtain the thermal fluctuations as a function of tip-substrate distance is shown in Fig. 1(a). While ramping up and down the substrate close to the tip as slow as possible, we monitor the fluctuations of the

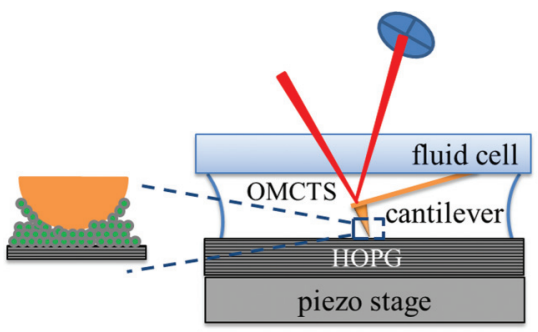

(a)

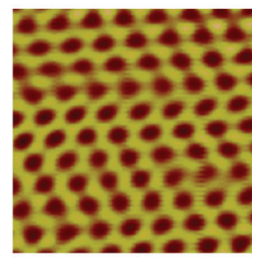

(b)
FIG. 1. (Color online) (a) Schematic representation of the experimental setup. (b) Friction microscopy image of HOPG $(2 \times 2 \mathrm{~nm})$ after Fourier filtering. deflection signal. As a substrate we use freshly cleaved and atomic flat highly oriented pyrolytic graphite (HOPG) with an in-plane lattice constant of $0.25 \mathrm{~nm}$ (Mikromasch grade ZYB). For the liquid we chose octamethyl-cyclotetrasiloxane (OMCTS, as received from Fluka, purum $\geqslant 99.0 \%$ ) because its molecules have a relatively large diameter of $0.8 \sim 0.9 \mathrm{~nm}$. The AFM device itself is a Veeco Multimode 8 with a Nanoscope $\mathrm{V}$ controller and Veeco EV scanner. The AFM is housed in an acoustic isolation box and operated at a constant temperature of $300 \mathrm{~K}$. For the nanoconfinement measurements, cantilevers with a sharp tip were used, from Mikromasch, which were NSC36 aluminum coated on the back side. They have a spring constant of $k_{c}=1 \sim 4 \mathrm{~N} / \mathrm{m}$, as determined in air using the thermal calibration method [42], and a resonance frequency $f_{r}$ in liquid between 45 and $80 \mathrm{kHz}$, as determined $8 \mathrm{~nm}$ away from the substrate. For the Reynolds damping measurements, cantilevers with a relatively blunt silicon tip were used, from Team nanotech, which were LRCH coated with aluminum on the back side. These have a $k_{c}$ of $\sim 2 \mathrm{~N} / \mathrm{m}$ and a $f_{r}$ in liquid of $\sim 25 \mathrm{kHz}$. Prior to the experiments cantilevers and fluid cell are rinsed in isopropanol and ethanol, after which the cantilevers are treated for $1 \mathrm{~min}$ in a plasma cleaner (Harrick Plasma). After the measurements the tips are characterized by high-resolution scanning electron microscopy (HR-SEM Zeiss LEO 1550) to estimate the tip radius and to make sure that the cantilevers were clean [55]. The sharp tips turned out to have radii of $30 \sim 70 \mathrm{~nm}$ and the blunt tips $\sim 900 \mathrm{~nm}$.

\section{B. Experimental procedures}

As stated above, two kinds of experiments are conducted: one is done with blunt tips to validate the thermal noise approach by measuring the Reynolds damping and the other is done with sharp tips to probe the dynamics in the layered liquid. The experimental procedures are identical, but the experimental parameters differ slightly.

The AFM is operated in force-distance mode. While the distance between cantilever tip and substrate is varied periodically at low speed, the deflection signal $z(t)$ is monitored at a sampling rate of $500 \mathrm{kHz}$ (i.e., over 6 times the cantilever's fundamental eigenfrequency) using a low-pass filter with a bandwidth of $200 \mathrm{kHz}$ to prevent aliasing. To correct for the drift of the piezo stage, due to thermal expansion or creep, we use a fixed $(2 \sim 4 \mathrm{~nm})$ maximum deflection of the cantilever as set point for the highest position of the stage, i.e., as retraction threshold, after which the stage is retracted backwards over a fixed distance before the next approach is started. From the variations in the approach distances we estimate that the drift during the measurements is always less than $160 \mathrm{pm} / \mathrm{s}$. Therefore, we choose an approach speed of $1 \mathrm{~nm} / \mathrm{s}$ and a ramp size of $10 \mathrm{~nm}$ for the measurements with the sharp tips. During measurements with blunt tips, i.e., the Reynolds damping measurements, the retraction speed, distance, and threshold are $8 \mathrm{~nm} / \mathrm{s}, 25 \mathrm{~nm}$, and $4 \mathrm{~nm}$, respectively. In both cases, these parameters guarantee data acquisition time at acceptable drift.

In the case of sharp tips, the retraction threshold of $2 \mathrm{~nm}$ ensures hard contact between the tip and the sample, i.e., all OMCTS will be squeezed out. This is concluded from friction force images recorded at the same deflection set point, which corresponds to a load of $\sim 5 \mathrm{nN}$. As shown in Fig. 1(b), these 
data reveal a hexagonal lattice (lattice constant of $0.25 \mathrm{~nm}$ ) characteristic for HOPG.

\section{Model}

Although the SHO model is not fully appropriate to describe the cantilever dynamics over a wide frequency range, see Sec. V, and the Appendix for discussion of this issue, the deviation from the full solution is only a few percentages within our fitting range, so this approach is more than sufficient. The SHO is driven by the Brownian force along with tip-substrate interactions,

$$
m^{*} \ddot{z}+\gamma_{c} \dot{z}+k_{c} z=F_{\mathrm{ts}}+F_{B}(t),
$$

where $z$ is the tip position, $m^{*}$ the total effective mass (including the added mass originating from the motion of the surrounding liquid), $\gamma_{c}$ the viscous damping around the cantilever, $k_{c}$ the intrinsic cantilever stiffness, $F_{\text {ts }}$ the distancedependent tip-substrate interaction, and $F_{B}$ the random force due to Brownian motion, characterized by $\left\langle F_{B}\right\rangle=0$ and $\left\langle F_{B}(s) F_{B}(s+t)\right\rangle=2 \gamma k_{B} T \delta(t)$, where $k_{B} T$ is the thermal energy and $\delta(t)$ the Dirac $\delta$ function. According to the equipartition theorem, the average potential energy of the cantilever, $\frac{1}{2} k\left\langle z^{2}\right\rangle$, is equal to $\frac{1}{2} k_{B} T$, so for a cantilever stiffness of $2 \mathrm{~N} / \mathrm{m}$ the root-mean-square displacement of the unperturbed thermal motion is around $46 \mathrm{pm}$ at room temperature. This amplitude is much smaller than characteristic length scale of the variations in the tip-substrate interaction, which for OMCTS is about $0.9 \mathrm{~nm}$. Therefore, linearization of the tip-substrate force around the average tip displacement is justified, i.e., $F_{\text {int }}=$ $F_{0}-k_{\text {int }} z-\gamma_{\text {int }} \dot{z}$, where $k_{\text {int }}$ is the interaction stiffness and $\gamma_{\text {int }}$ is the interaction damping, and Eq. (1) can be rewritten as

$$
m^{*} \ddot{z}+\gamma \dot{z}+k z=F_{B}(t),
$$

where $z$ is now the tip displacement with respect to its average position at distance $d, \gamma=\gamma_{c}+\gamma_{\text {int }}$ is the total damping coefficient, and $k=k_{c}+k_{\text {int }}$ the total stiffness. Solving Eq. (2) in the frequency domain, we get

$$
z[\omega]=\left(k-m^{* 2}+j \gamma \omega\right)^{-1} F_{B}[\omega],
$$

where $j=\sqrt{-1}$ is the imaginary unit. The PSD of the displacement $z$ is defined as

$$
P_{z z}(\omega)=\lim _{t_{s} \rightarrow \infty}\left(\frac{1}{t_{s}} z[\omega] z[\omega]^{*}\right),
$$

where $t_{s}$ is the sampling time. According to the WienerKhinchin theorem [51,52], the PSD of the Brownian force $F_{B}$ is related to its ACF as $P_{F F}(\omega)=2 \gamma k_{B} T$. Hence, $P_{z z}(\omega)$ can be rewritten as

$$
P_{z z}(\omega)=P_{w}+\frac{P_{o}}{\left[1-\left(\frac{\omega}{\omega_{r}}\right)^{2}\right]^{2}+\left(\frac{\omega}{\omega_{r} Q}\right)^{2}},
$$

where $P_{w}$ accounts for the instrumental (white) noise, $\omega_{r}$ is the resonance frequency, $Q$ the quality factor, and $P_{o}$ a scaling factor. Both $Q$ and $\omega_{r}$ depend on tip-substrate distance and are related to $k_{\text {int }}$ and $\gamma_{\text {int }}$ by

$$
\begin{aligned}
& k=k_{\text {int }}+k_{c}=m^{*} \omega_{r}{ }^{2}, \\
& \gamma=\gamma_{\text {int }}+\gamma_{c}=m^{*} \omega_{r} / Q .
\end{aligned}
$$

The effective mass $m^{*}$ is estimated by substituting the intrinsic cantilever stiffness $k_{c}$ and the resonance frequency $\omega_{r}$, as measured in liquid away from the substrate, where the tipsubstrate interaction can be neglected, into Eq. (2). As stated before, the stiffness $k_{c}$ itself is obtained from the thermal calibration procedure in air.

Another way to determine the interaction parameters is to measure the ACF of the displacement signal $z(t)$, defined as

$$
R_{z z}(t)=\frac{1}{t_{s}} \int_{0}^{t_{s}} z(s) z(s+t) d s
$$

Since $R_{z z}(t)$ is equal to the inverse Fourier transform of the PSD, for $Q>0.5$ one can show that [56]

$R_{z z}(t)=\frac{k_{B} T}{m^{*} \omega_{r}^{2}} \exp \left(\frac{-\omega_{r} t}{2 Q}\right)\left[\cos \omega_{1} t+\frac{\sin \omega_{1} t}{\sqrt{(2 Q)^{2}-1}}\right]+R_{0}$,

where $\omega_{r}=\sqrt{k / m^{*}}, \omega_{1}=\omega_{r} \sqrt{\left(1-(2 Q)^{-2}\right)}$, and $R_{0}$ a constant to compensate for a possible background. By fitting Eq. (7) to the measured ACF one again obtains values for $Q$ and $\omega_{r}$.

\section{DATA ANALYSIS}

After we have recorded the tip displacement as a function of time during ramping, this time sequence $z\left(t_{n}\right)$ is split into small time intervals, each of which contains $2^{16}$ data points. From the displacement versus time sequence in each interval the background, i.e., best-fitting straight line, is subtracted before the time sequence is transformed into a power spectral density, Eq. (3), using a standard fast-Fourier-transform algorithm to calculate $z[\omega]$. Next, our model [Eq. (4)] is fitted to the obtained $P_{z z}(\omega)$, revealing values for $k_{\text {int }}(d)$ and $\gamma_{\text {int }}(d)$, where $d$ is the average tip-substrate distance during the considered time interval. Alternatively, the ACF of the time sequence is calculated with Eq. (6) and our model function [Eq. (7)] is fitted to this correlation, again resulting in best-fit values for $k_{\text {int }}(d)$ and $\gamma_{\text {int }}(d)$.

Although this analysis is straightforward, one has to keep in mind two aspects. First, the accuracy of the obtained values for $Q$ and $\omega_{r}$ depends strongly on $Q$ and the number of data points $N$ in the time sequence. For $N=2^{16}$ and $Q>1.5$ we obtain $\Delta Q / Q<0.1$ and $\Delta \omega_{r} / \omega_{r}<0.01$ during fitting. The accuracy is further improved by averaging over several, typically 50, approach curves. For $Q<1$ the system is overdamped and the uncertainty in both $Q$ and $\omega_{r}$ strongly increases with decreasing $Q$ value. In the case of sharp tips in our experiments, this occurs at $d<1.5 \mathrm{~nm}$. Hence, the method gives reliable results for distances larger than $1.5 \mathrm{~nm}$. Second, during the sampling of a time sequence the substrate is not stationary but travels $0.13 \mathrm{~nm}$; the tip-substrate distance changes between 0.07 and $0.24 \mathrm{~nm}$ depending on the tip-substrate interaction at that distance. In all cases, this variation is sufficiently small compared to the diameter of the OMCTS molecules, i.e., the length scale on which the tip-substrate interaction is expected to vary. 


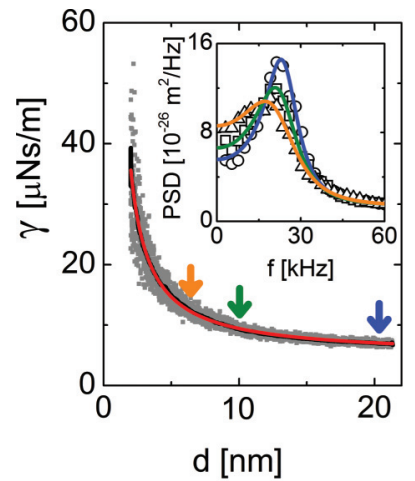

(a)

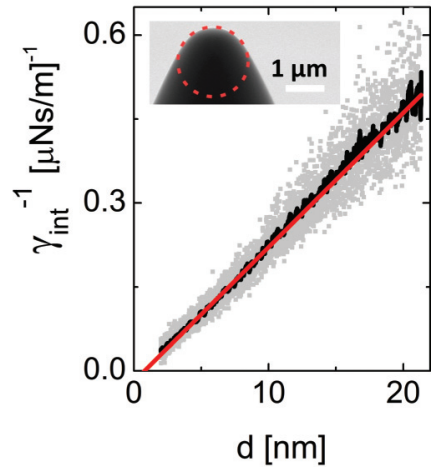

(b)
FIG. 2. (Color online) (a) Damping coefficient versus tipsubstrate distance extracted from 25 approaches. Black line: averaged data, using a 25-point moving average. Red curve: best fit [Eq. (8)] to the data for damping coefficient. The inset shows the measured (smoothed) PSD (symbols) at three different distances denoted by the arrows in the main graph (solid lines are fits to our model function). (b) Inverse of Reynolds damping coefficient as a function of tip-substrate distance. Black line: averaged data, using a 25-point moving average. Red line: linear fit curve. The inset shows the SEM image of the tip used in the experiments $(R=900 \mathrm{~nm}$, red circle). Parameter values of cantilever: $k_{c}=1.91 \pm 0.06 \mathrm{~N} / \mathrm{m}$; $f_{r}=\omega_{r} / 2 \pi=25.2 \pm 0.3 \mathrm{kHz}$ in liquid at $d \approx 21 \mathrm{~nm}\left(m^{*}=7.61 \times\right.$ $10^{-11} \mathrm{~kg}$ ).

\section{RESULTS}

We first discuss the validation of our method using the Reynolds damping measurements with blunt tips of radius $R_{\text {tip }} \approx 900 \mathrm{~nm}$. The inset of Fig. 2(a) gives three exemplary power spectra with best-fit curves at various distances indicated by the (color matched) arrows in the main panel. The spectra are extracted from 25 approach curves, measured consecutively with the same tip. As the distance decreases, the peaks in the power spectra become broader, i.e., the fitted $Q$ values become smaller, and the resonance shifts towards lower frequencies. From the fitted $Q$ values we calculate the damping coefficient using Eq. (5b). The total damping increases monotonically with decreasing tip-substrate distance. The expected hydrodynamic Reynolds damping due to the confined liquid under the tip is given by

$$
\gamma(d)=\gamma_{c}+6 \pi \eta \frac{R_{\mathrm{tip}}^{2}}{d-\Delta},
$$

where $\gamma_{c}$ is the damping experienced by the cantilever beam and $\Delta$ is an offset to compensate for the error in zero separation. $\eta$ is the viscosity of the liquid.

To ensure bulk behavior, we exclude data at distances less than $5 \mathrm{~nm}$ from the fit. This lower limit is obtained from the results of measurements with a sharp tip (see below). Fitting Eq. (8) to our data, we find a viscosity $\eta=2.7 \pm 0.2 \mathrm{mPa} \mathrm{s}$, in agreement with literature data: $2.2 \sim 2.5 \mathrm{mPa}$ s [24,57]. For $\Delta$ we obtain a value of about $0.8 \mathrm{~nm}$, which indicates that an equivalent of one molecular layer remains rigidly stuck to one of the two the solid surfaces in qualitative agreement with earlier SFA measurements [6,14]. To examine the quality of the fit, the inverse of the Reynolds damping has been plotted versus the tip-substrate distance in Fig. 2(b). The inverse damping indeed shows a linear behavior, down to the smallest tip-substrate distances [58]. From this observation we conclude that the choice of the lower bound of $5 \mathrm{~nm}$ in our analysis is not critical and that slip is absent in our system (as expected for a complete wetting system).

We also validated the conservative tip-substrate forces by extracting the force gradient from the distance-dependent resonance frequency. For separations larger than $9 \mathrm{~nm}$, we find a monotonically increasing attraction that can be described by van der Waals interaction between the tip and graphite substrate across the liquid film (see Fig. S1 in the Supplemental Material [59]). At $d<6 \mathrm{~nm}$, the attraction becomes repulsive, with a weak oscillatory component superimposed. While the oscillation has the expected periodicity corresponding to molecular diameter, the features are not very pronounced, presumably due to the poorly defined geometry of the large tips on small scales.

Overall, these validation measurements demonstrate that our method does indeed yield quantitatively correct values for the forces, including in particular dissipative forces down to a few nanometers of tip-substrate separation. They provide a crucial link between well-established continuum physics and the nanoscale behavior to be described below, which has proven difficult to achieve in many earlier studies of confined liquids.

In Figs. 3-5 we present the tip-substrate interactions measured with a sharp tip close to the substrate. The main panel of Fig. 3 shows the average deflection of the cantilever during approach up to the retraction threshold of $2 \mathrm{~nm}$. The insets show the measured PSDs (right) and ACFs (left) at three different distances indicated by the (color-matched) arrows in the main graph. The solid lines represent our model fits [Eqs. (4) and (7)] from which we extract the values of $\omega_{r}$ and $Q$. At distances corresponding to positive (negative) force gradients, as denoted by an olive (blue) arrow, the resonance

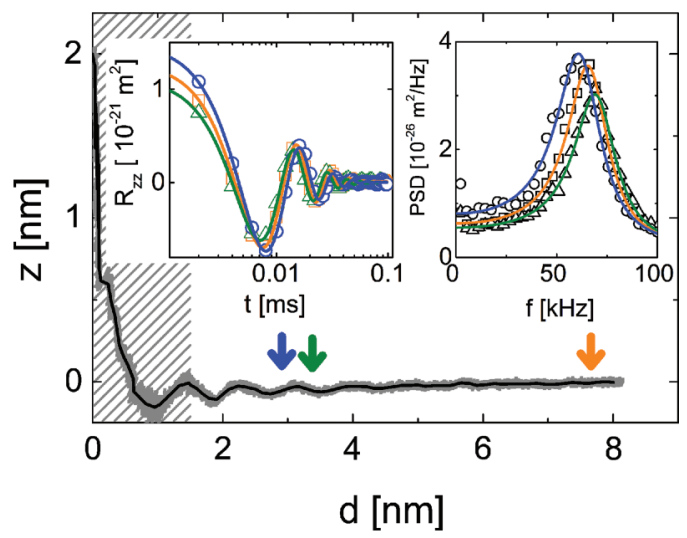

FIG. 3. (Color online) Measured cantilever deflection (gray) upon approach of the tip towards the surface at $1 \mathrm{~nm} / \mathrm{s}$ (black line, averaged data, using a $2^{16}$-point moving average). The inset shows the measured autocorrelations $R_{z z}$ and measured (smoothed) power spectrum density PSDs (symbols) at three different distances denoted by the arrows in the main graph (solid lines are fits to our model function). Data at $d<1.5 \mathrm{~nm}$ are shadowed, because they are not taken into account in further analysis; see Figs. 4 and 5. Cantilever parameters: $R_{\text {tip }}=45 \mathrm{~nm}, k_{c}=2.71 \pm 0.08 \mathrm{~N} / \mathrm{m} ; f_{r}=67.5 \pm 0.2 \mathrm{kHz}$, and $Q=2.82 \pm 0.08$ at $d=8 \mathrm{~nm}\left(m^{*}=1.51 \times 10^{-11} \mathrm{~kg}\right)$. 


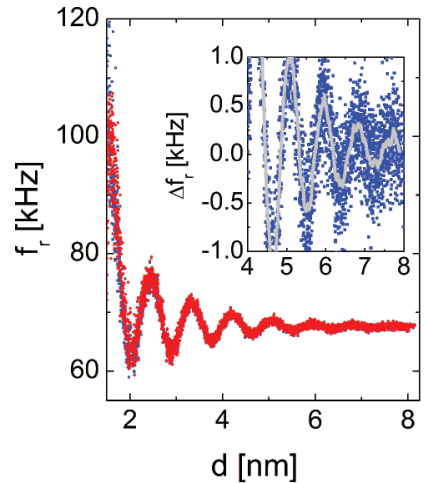

(a)

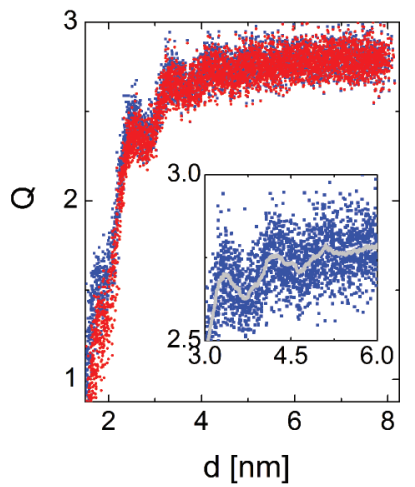

(b)
FIG. 4. (Color online) Fitted resonance frequency (a) and quality factor (b) versus tip-substrate distance extracted from 97 approaches; blue squares denote the PSD analysis and red dots denote the ACF analysis. (a) The inset shows the resonance frequency shifts and also the averaged one extracted by PSD analysis. (b) The inset shows the fitted quality factor and the averaged one by PSD analysis.

frequency is shifted towards larger (smaller) frequencies. Correspondingly, the magnitude of the autocorrelation function is reduced (increased) and the position of the first zero crossing is shifted towards shorter (larger) times, in agreement with our expectations. For values of $d<1.5 \mathrm{~nm}$, the cantilever motion becomes overdamped and the fits to both the PSD and ACF no longer yield reliable values.

We note here that the PSDs shown in Fig. 2(a) and Fig. 3 display a small excess power at low frequencies compared to the SHO fit. At the same time, we slightly shift the ACFs vertically to make them decay to zero for long times. We will explain the origin of these effects in the discussion section.

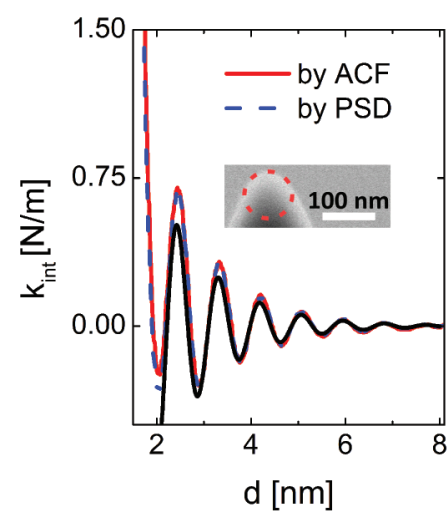

(a)

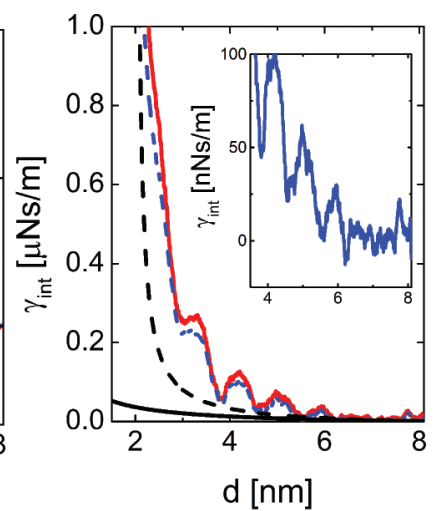

(b)
FIG. 5. (Color online) Interaction stiffness (a) and damping (b) versus distance. Blue lines: results from the PSD analysis; red lines: results from the ACF analysis. Both are smoothed using a 97-point moving average. (a) Solid black line: best fit of the oscillatory decaying stiffness profile. The inset shows an SEM image of the tip. (b) Solid black line: calculated Reynolds damping, using the reference bulk viscosity and the tip radius obtained from the SEM image. Dashed black line: obtained by shifting the black solid line $2 \mathrm{~nm}$ to the right. Both calculated lines are offset vertically so they are aligned to experimetnal data at $8 \mathrm{~nm}$. The inset shows a close-up of the average damping coefficient obtained from the PSD analysis.
In Fig. 4, we plot the values for (the shift in) the resonance frequency $\omega_{r}$ and the quality factor $Q$ obtained from the analysis the PSD and the ACF as a function of $d$. The data represent an average of 97 approach curves. The individual curves were aligned by shifting the two peak positions at $\sim 2.4 \mathrm{~nm}$ and $\sim 3.3 \mathrm{~nm}$ in the resonance frequency profile (see Fig. 4) on top of each other to compensate for residual drift. The offset per curve is typically smaller than $0.1 \mathrm{~nm}$ (see Fig. S2 in the Supplemental Material [59]). Figure 4 clearly shows that the results from the ACF and PSD analyses are in perfect agreement. Both methods are, thus, equally viable and allow for detecting oscillatory solvation forces up to distances of at least $8 \mathrm{~nm}$, as the variation of the resonance frequency shows. The inset of Fig. 4(a) suggests that we can detect variations of the resonance frequency down to values of $\Delta f \approx 50 \mathrm{~Hz}$.

In Fig. 5, we show the interaction stiffness $k_{\text {int }}(d)$ and the interaction damping $\gamma_{\text {int }}(d)$ as extracted from data in Fig. 4 using Eq. (5) (assuming $\gamma_{\text {int }}=0$ at $d=8 \mathrm{~nm}$ ). The shape of the conservative solvation force gradient is well described by Refs. [8] and [10] as follows:

$$
k_{\text {int }}(d)=K_{\text {int }} \exp \frac{-(d+\Delta d)}{\epsilon} \cos \left[\frac{2 \pi(d+\Delta d)}{\sigma}\right] .
$$

Here $\sigma=0.88 \mathrm{~nm}$ is the periodicity determined directly from the oscillatory profile; the scaling factor $K_{\text {int }}$, the decay length $\epsilon$, and the offset $\Delta d$ are obtained from the best fit. Data at a distance less than $2 \mathrm{~nm}$ are excluded from the procedure, because the uncertainty in $f_{r}$ and $Q$ is too large. The black curve in Fig. 5(a) is the best-fit profile with $K_{\text {int }}=3.6 \pm$ $0.1 \mathrm{~N} / \mathrm{m}, \epsilon=1.39 \pm 0.02 \mathrm{~nm}$, and $\Delta d=0.194 \pm 0.001 \mathrm{~nm}$. To convert the measured interaction stiffness $k_{\text {int }}$ to a normalized interaction force $F / R$ we integrate the local oscillatory pressure $p(x)=-p_{\infty} \cos (2 \pi x / \sigma) \exp (-x / \epsilon)$ using the Derjaguin approximation [10] to obtain $F(d)=2 \pi R \int_{d}^{\infty} p(x) d x$, where $x(r)=d+r^{2} /(2 R)$. From this calculation we obtain an amplitude $F / R=9 \pm 3 \mathrm{mN} / \mathrm{m}$, where the tip radius $R$ is characterized by SEM imaging [see SEM image of the tip after use in Fig. 5(a)]. The periodicity $\sigma$, the decay length $\epsilon$, and the normalized interaction force $F / R$ are in good agreement with earlier measurements using SFA or AFM [8,24].

The interaction damping features clear oscillations superimposed onto an overall increasing background, as shown in Fig. 5(b). Local maxima and minima in the dissipation can be clearly distinguished between $d=2 \mathrm{~nm}$ and $6 \mathrm{~nm}$, i.e., over a much larger range than reported previously [24]. The location of the local maxima coincides with the maxima in the interaction stiffness in Fig. 5(a).

The absolute value of the dissipation is approximately 10 times higher than the expectation based on the prediction of Reynolds theory, i.e., continuum hydrodynamics in combination with the bulk viscosity and $\Delta=0$ in Eq. (8), which is shown as a solid line in Fig. 5(b). If we follow earlier suggestions $[9,14]$ to include a stagnant layer of finite thickness, the thus-corrected continuum model comes closer to the experimental data, as exemplified by the dashed line in Fig. 5, which corresponds to $\Delta=2 \mathrm{~nm}$. Therefore, it is worth noting that for comparing the experimental data to the continuum model, it is critical to take into account the (non-)existence of stagnant layers. 


\section{DISCUSSION}

Before we discuss our results, also in relation to earlier studies, we first consider the advantages and disadvantages of the thermal noise approach in more detail.

\section{A. Approximating the cantilever as an SHO}

Although in the SHO approach the cantilever is modeled as a point mass attached to a massless spring, it is rather accurate in describing the lowest vibrational mode of a cantilever [60] because, in the lowest oscillation mode, all parts of the cantilever oscillate perfectly in phase with the tip. Hence, effective values for the mass $m^{*}$, damping $\gamma_{c}$, and stiffness $k_{c}$ of the cantilever, which can all be determined by calibration measurements, are sufficient to describe the cantilever dynamics. However, the cantilever bending depends also weakly on the external load on the tip. This causes a small systematic error. Yet, a decomposition of the cantilever into small segments for typical parameter values $(Q \sim 3)$ shows that the relative error in the interaction parameters $k_{\text {int }}$ and $\gamma_{\text {int }}$ with respect to $k_{c}$ and $\gamma_{c}$ is never larger than typically $3 \%$ for $\left|k_{\text {int }} / k_{c}\right|<0.9$ and $\left|\gamma_{\text {int }} / \gamma_{c}\right|<0.8$ (see also the Appendix). The experimental error in the interaction stiffness and damping coefficient, after averaging over typically 50 approach curves, is less than $1 \%$ and smaller than the systematic error but of the same order of magnitude. If needed, the deviations presented in the Appendix can be used as correction factors.

\section{B. Influence of noise and approach speed}

The cantilever displacement recorded as a function time has been analyzed using both its PSD (in the frequency domain) and its ACF (in the time), leading to identical results within experimental error. The obtained PSDs (see Figs. 2 and 3) show indeed no spurious resonance peaks as observed in the case of actively driven cantilevers. However, a small white noise background is observed, as well as an excess power at low frequencies $(<10 \mathrm{kHz})$. This low-frequency excess noise power is distance dependent and, hence, originates only partially from the electronic $1 / f$ noise. A second contribution can be attributed to the finite sampling time in combination with the approach speed of the substrate. During the sampling time for each data block ( $2^{16}$ points), the substrate is displaced over $0.13 \mathrm{~nm}$. This displacement leads to a variation of the tip-substrate interaction and, hence, to a variation of the average tip position of at most $0.1 \mathrm{~nm}$ per acquisition, which affects the calculation of the PSD. This effect can be reduced by reducing the sampling time. Experimental tests show indeed a strong reduction of this low-frequency excess noise power. However, at the same time, the accuracy decreases. These findings were confirmed by numerical simulations. In the time domain the white noise contribution to the signal is only detected at zero time lag while the power excess at low frequencies manifests itself by an offset in the ACF. These findings were also confirmed by numerical simulation. In both approaches, the effects can be fully taken into account, without introducing any systematic error into the analysis.

\section{Comparison with acoustic or magnetic driving}

As mentioned above, we observe no spurious resonance in our PSDs, which can hardly be prevented in acoustic actuation
[26,33]. Moreover, because the thermal noise covers a broad frequency range, it is sufficient to determine the PSD or ACF, circumventing the tedious phase determination as necessary in acoustic or magnetic driving. These spurious resonances and possible errors in the phase determination give rise to an erroneous coupling of the conservative and dissipative contributions in the analysis of the tip-substrate interactions.

Overall, we can state that the thermal noise method is more sensitive than methods proposed so far as long as the quality factor is greater than 1. The detection threshold for the resonance frequency shift is $50 \mathrm{~Hz}$ at $70 \mathrm{kHz}$, while variations in $Q$ of $0.5 \%$ are detectable after averaging over typically 50 approach curves (see Fig. 4). For $Q<1$, i.e., for small tip-substrate distances, the method cannot be used. However, by optimizing the cantilever geometry [61], the $Q=1$ condition is reached for smaller distances.

\section{Reynolds damping measurements}

Measuring the Reynolds damping in the distance range from 5 to $20 \mathrm{~nm}$, we were able to validate the method as can be concluded from Fig. 2. Indeed, the expected $d^{-1}$ dependence was found as well as the right bulk viscosity of OMCTS at room temperature if we allow for a small offset in the apparent tip-substrate distance of $0.8 \mathrm{~nm}$. We attribute this offset to a combination of possible local irregularities on the tip surface $[62,63]$ and an uncertainty in our exact "zero" tip-substrate distance (in contrast to the measurements with the sharp tip, we were unable to image the graphite lattice with these blunt tips). Thus, within the experimental accuracy, no slip of the OMCTS on the HOPG substrate is observed, in line with complete wetting of OMCTS on HOPG. Alternatively, the offset can be attributed to the existence of an immobile layer on the substrate causing a negative slip length (the zero-velocity plane is shifted inwards). Negative slip lengths have been reported in SFA studies on OMCTS confined between two mica sheets $[9,14]$ and can, in general, be caused by commensurability between the solid and the liquid [64]. Globally, OMCTS and graphite are incommensurate. Yet it was recently shown in MD simulations that specific chemical interactions [65] or an interaction-induced geometric adjustment of the atoms in the liquid-molecule to the surface [66] can also result in a negative slip length. However, there has been no direct evidence of these effects occurring between OMCTS and graphite [67].

\section{E. Solvation force and distance-dependent dissipation}

The results obtained with sharp tips for tip-substrate distances ranging from 2 to $8 \mathrm{~nm}$ show clearly the decaying oscillatory behavior of the stiffness due to the conservative solvation force versus distance. The thermal noise method is significantly more sensitive than earlier measurements using acoustically driven cantilevers [24]. While those earlier measurements revealed excess dissipation only on squeezing out the last three layers of OMCTS up to a maximum tipsubstrate separation of approximately $d=2.4 \mathrm{~nm}$, the current measurements reveal an oscillatory structure in the dissipation up to $d=6 \mathrm{~nm}$ with a total of five local maxima. Yet, the thermal noise method cannot probe the liquid layers in immediate contact with the solid surface due to excessive damping for 
$d<1.5 \mathrm{~nm}$. According to Fig. 3, this implies that the local maxima in the dissipation shown in Fig. 5(b) correspond to film thicknesses of three to seven molecular layers. The local maximum at the smallest tip-substrate separation shown in Fig. 5(b) (at $d \approx 2.2 \mathrm{~nm}$ ) thus corresponds to the peak at the largest tip-substrate separation in Refs. [24,54] at $d \approx 2.4 \mathrm{~nm}$. (The absolute zero of the tip-substrate separation in Ref. [24] is off by approximately $0.6 \mathrm{~nm}$.) This assignment is corroborated by the absolute value of $\gamma_{\text {int }}$, which is approximately $10^{-6} \mathrm{Ns} / \mathrm{m}$ in both experiments. Comparing the sensitivity of the two experiments reveals a gain of approximately one order of magnitude in the present experiments.

The MD simulations reported in Ref. [54] suggest that the sharp excess dissipation on squeezing out the layers in immediate contact with the solid walls is related to confinement-induced transitions between very well ordered (solidlike) films for film thicknesses of one, two, and perhaps three molecular layers and rather disordered configurations for intermediate half-integer numbers of molecular layers. Whether that scenario also extends to the much larger tipsubstrate separations studied here is not clear. At larger separations, thermal fluctuations as well as the tip geometry on a larger scale may lead to increased disorder [68-70] and, thus, prevent the crystalline arrangement found at smaller separations.

\section{CONCLUSION}

In summary, we measured the distance-dependent solvation forces and the dissipation of OMCTS in the confinement between a silicon tip and an HOPG substrate using atomic force microscopy. To obtain reliable results for the distancedependent dissipation in the confinement, we employ the thermal noise approach, which provides a resolution of approximately $50 \mathrm{~Hz}$ in frequency shift and $10 \mathrm{nNs} / \mathrm{m}$ in damping coefficient as long as the quality factor is larger than unity, which is the case for tip-substrate distances larger than $2 \mathrm{~nm}$ in the present experiments. To validate the method the distance-dependent Reynolds damping and bulk viscosity of OMCTS were successfully measured. Close to the substrate we were able to measure the interaction stiffness due to the solvation forces in agreement with earlier findings, while the damping also showed pronounced oscillations instead of monotonic behavior as a function of tip-substrate distance. From a technical perspective, we presented a method to perform small amplitude force microscopy without relying on a perfect spectral response, in both amplitude and phase, to the external actuation of the cantilever, just by analyzing the thermal noise of the cantilever, which also guarantees small amplitudes of typically $50 \mathrm{pm}$. Moreover, it was shown that the thermal noise can be evaluated equally well with the power spectral density as with the autocorrelation function.

\section{ACKNOWLEDGMENTS}

This work has been supported by the Foundation for Fundamental Research of Matter (FOM), which is financially supported by the Netherlands Organization for Scientific Research (NWO).

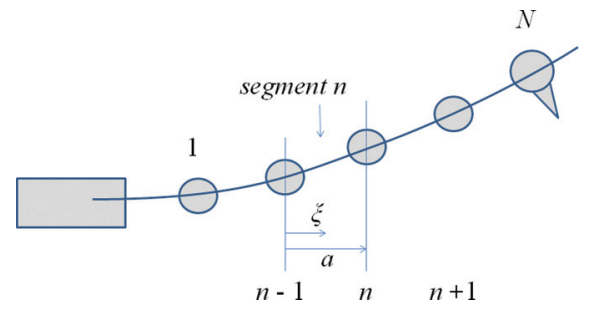

FIG. 6. (Color online) Sketch of the configuration considered.

\section{APPENDIX: THERMAL VIBRATIONS OF A CANTILEVER BEAM WITH TIP-SUBSTRATE INTERACTION}

As stated in Sec. II C, we model the cantilever dynamics as a SHO with only one resonance frequency. However, due to its distributed mass and friction, the cantilever will show several vibrational bending modes, each with its own resonance frequency. In our experimental approach we consider only the frequency response around the lowest (and most prominent) resonance frequency. In this Appendix we will show that in this frequency regime the cantilever behavior deviates only slightly from SHO behavior also in the presence of an interaction force at the tip of the cantilever. Moreover, we will quantify these deviations in terms of the interaction stiffness and interaction damping as defined in Sec. II C by comparing the full frequency response with that of a best-fitting SHO response.

We consider the cantilever as a chain of $N$-distributed masses connected by massless cantilever segments as depicted in Fig. 6. Bead $n$ with mass $m$ exerts a force $B_{n}$ on the cantilever which is equal to

$$
B_{n}=f_{n}-m \ddot{u}_{n}-\zeta \dot{u}_{n},
$$

where $f_{n}$ is the external (in this case Brownian) force on bead $n, u_{n}$ its displacement, and $\zeta$ its friction coefficient. If the tip interacts with the substrate we replace $f_{N}$ by $f_{N}-\left(k_{\text {int }} u_{N}+\left(\gamma_{\text {int }}+\zeta_{\text {tip }}\right) \dot{u}_{N}+m_{\text {tip }} \ddot{u}_{N}\right)$.

Assuming $N$ beads on the cantilever, with spacing $a$, we consider a single segment $n$ situated between $m_{n-1}$ and $m_{n}$. Both the total force $F$ and torque $S$ should be zero on this segment:

$$
\begin{gathered}
F_{r}^{[n]}-F_{l}^{[n]}=0, \\
S_{r}^{[n]}+a F_{r}^{[n]}-S_{l}^{[n]}=0,
\end{gathered}
$$

where $r$ stands for right and $l$ for left.

$$
\begin{gathered}
\text { Since } F_{r}^{[n]}=B_{n}+F_{l}^{[n+1]} \text { and } S_{r}^{[n]}=S_{l}^{[n+1]} \text { we define } \\
F_{n}=F_{r}^{[n]} \text { and } S_{n}=S_{r}^{[n]}
\end{gathered}
$$

and Eq. (A3) reduces to

$$
S_{n}+a F_{n}=S_{n-1} .
$$

The force $F_{n}$ is equal to the sum of all external forces $B_{m}$ acting on the right of segment $n$,

$$
F_{n}=\sum_{m=n}^{N} B_{m},
$$


while the torque $S_{n}$ is equal to the sum of all external moments $(m-n) a B_{m}$ acting on the right of segment $n$,

$$
S_{n}=a \sum_{m=n}^{N}(m-n) B_{m} .
$$

Solving the force and torque balance on segment $n$, one obtains relations for the displacement $u$ and the slope $a d u / d x$ of mass $n$ and $n-1$ as follows:

$$
\begin{gathered}
u_{n-1}=u_{n}-u_{n}^{\prime}+\frac{S_{n} / a}{2 \kappa}+\frac{F_{n}}{6 \kappa}, \\
u_{n-1}^{\prime}=u_{n}^{\prime}-\frac{S_{n} / a}{\kappa}-\frac{F_{n}}{2 \kappa}, \\
S_{n-1}=S_{n}+a F_{n}, \\
F_{n-1}=F_{n}+B_{n-1},
\end{gathered}
$$

where $\kappa=E I / a^{3}$ and $B_{n}=f_{n}-q u_{n}$ with $q(\omega)=-m \omega^{2}+$ $j \zeta \omega$. With Eqs. (A8)-(A11) we can calculate all $u_{n}$ and $u_{n}^{\prime}$ values in the frequency domain starting from a guess for $u_{N}$ and $u_{N}^{\prime}$ and we will end up with the following linear relations:

$$
\begin{aligned}
& u_{0}=A_{0} u_{N}+A_{1} u_{N}^{\prime}+\sum_{n=1}^{N} \alpha_{n} f_{n}, \\
& u_{0}^{\prime}=A_{2} u_{N}+A_{3} u_{N}^{\prime}+\sum_{n=1}^{N} \beta_{n} f_{n} .
\end{aligned}
$$

By inversion of the last two equations we can express $u_{N}$ and $u_{N}^{\prime}$ in $u_{0}, u_{0}^{\prime}$ and the forces $f_{n}$ accordingly:

$$
\begin{aligned}
& u_{N}=\frac{A_{3}}{[A]} u_{0}-\frac{A_{1}}{[A]} u_{0}^{\prime}+\sum_{n=1}^{N} G_{n} f_{n}, \quad G_{n}=\frac{\beta_{n} A_{1}-\alpha_{n} A_{3}}{[A]}, \\
& u_{N}^{\prime}=\frac{A_{0}}{[A]} u_{0}^{\prime}-\frac{A_{2}}{[A]} u_{0}+\sum_{n=1}^{N} H_{n} f_{n}, \quad H_{n}=\frac{\alpha_{n} A_{2}-\beta_{n} A_{0}}{[A]},
\end{aligned}
$$

with $[A]=A_{0} A_{3}-A_{1} A_{2}$. The coefficients $\alpha_{n}, \beta_{n}$, and $A_{n}$ can be calculated by evaluating Eqs. (A8)-(A11) while setting one of the values $\left(u_{N}, u_{N}^{\prime}, f_{1}, f_{2}, \ldots, f_{N}\right)$ equal to 1 and keeping all the other values 0 . Since we consider the noise response, we set both $u_{0}$ and $u_{0}^{\prime}$ to zero for all frequencies. Because the PSD of the Brownian forces is constant, i.e., $P_{f}(\omega)=2 \zeta k_{B} T$, the power spectral density $P_{S}(\omega)$ of the signal $u_{N}^{\prime}(\omega) / a$ is given by (no interaction on the tip)

$$
\begin{aligned}
P_{s}(\omega) & =\frac{1}{a^{2}} \sum_{n=1}^{N}\left|H_{n}(\omega)\right|^{2} P_{f_{n}}(\omega) \\
& =\frac{2 k_{B} T}{L^{2}} N^{2} \sum_{n=1}^{N} \zeta_{n}\left|H_{n}(\omega)\right|^{2} .
\end{aligned}
$$

Taking into account the interaction force (and additional tip mass) on the tip in the expression for $f_{N}$, we replace it

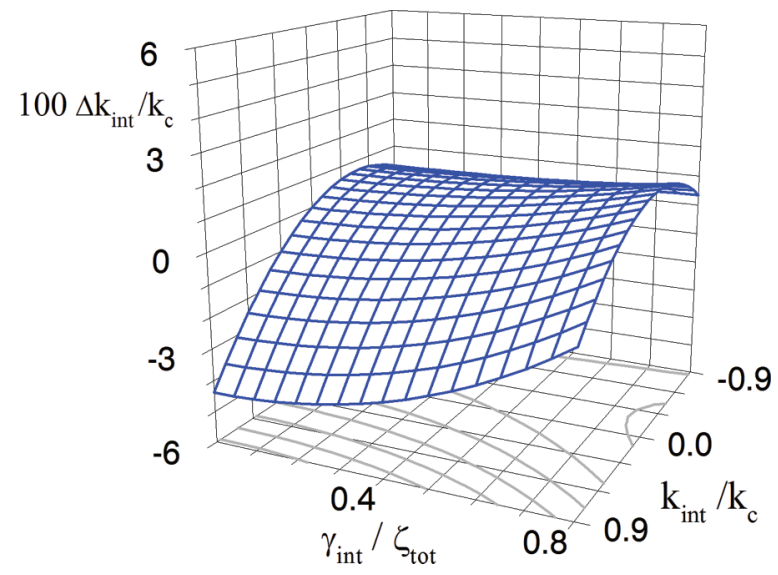

FIG. 7. (Color online) $\Delta k_{\text {int }}$ vs $\left(k_{\text {int }}, \gamma_{\text {int }}\right)$ for $N=32$ and $\alpha=1.0$. Note the vertical scale is blown up by a factor of $100 . k_{\text {int }}$ is taken relative to $k_{c}=3 E I / L^{3}$. The systematic error in $k_{\text {int }}$ is in all cases less than $4 \%$ of $k_{c}$.

by $f_{N}-\left(k_{\text {int }}+j \omega \gamma_{\text {int }}-m_{\text {added }} \omega^{2}\right) u_{N}$. Hence, we obtain, for our signal $u_{N}^{\prime}$,

$$
u_{N}^{\prime}=\sum_{n=1}^{N} H_{n} f_{n}-H_{N}\left(k_{\mathrm{int}}+j \omega \gamma_{\mathrm{int}}\right) u_{N}=\sum_{n=1}^{N} H_{n}^{[\mathrm{int}]} f_{n}
$$

with

$$
H_{n}^{[\mathrm{int}]}=H_{n}-\frac{H_{N}\left(k_{\mathrm{int}}+j \omega \gamma_{\mathrm{int}}\right)}{1+G_{N}\left(k_{\mathrm{int}}+j \omega \gamma_{\mathrm{int}}\right)} G_{n} .
$$

Because the interaction damping also modifies the random force on the tip, $P_{f_{N}}(\omega)=2 k_{B} T\left(\zeta_{N}+\gamma_{\text {int }}\right)$, the power spectral density of our signal becomes

$$
P_{S}(\omega)=\frac{2 k_{B} T}{a^{2}}\left(\left(\zeta_{N}+\gamma_{\text {int }}\right)\left|H_{N}^{[\mathrm{int}]}(\omega)\right|^{2}+\zeta \sum_{n=1}^{N-1}\left|H_{n}^{[\mathrm{int}]}(\omega)\right|^{2}\right) .
$$

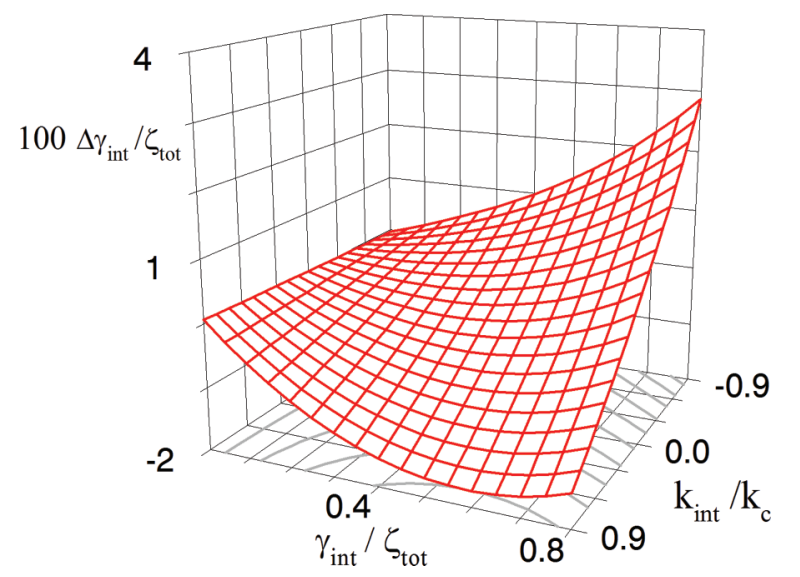

FIG. 8. (Color online) $\Delta \gamma_{\text {int }}$ vs $\left(k_{\text {int }}, \gamma_{\text {int }}\right)$ for $N=32$ and $\alpha=1.0$. Note the vertical scale is blown up by a factor of $100 . \gamma_{\text {int }}$ is taken relative to $\zeta_{\text {tot }}$. The systematic error in $\gamma_{\text {int }}$ is in all cases less than $3 \%$ of $\zeta_{\text {tot }}$. 
To evaluate Eq. (A18) and (A19) numerically, we define a reference frequency $\omega_{\#}=\sqrt{k_{c} / m_{\text {tot }}}$, where $k_{c}=$ $3 E I / L^{3}$. We then get, with $v=\omega / \omega_{\#}$, for the relevant variables,

$$
\begin{gathered}
q=-m \omega_{\#}^{2} \nu^{2}+j \zeta \omega_{\#}, \\
m=\alpha m_{\mathrm{tot}} / N, \\
m_{N}=(1-\alpha+\alpha / N) m_{\mathrm{tot}}, \\
\zeta=\alpha \zeta_{\mathrm{tot}} / N, \\
\zeta_{N}=(1-\alpha+\alpha / N) \zeta_{\mathrm{tot}},
\end{gathered}
$$

where $(1-\alpha) m_{\text {tot }}$ is the mass of the tip. With $Q^{-1}=$ $\zeta_{\text {tot }} / \sqrt{m_{\text {tot }} k_{c}}$ we arrive at

$$
q=\frac{\alpha k_{c}}{N}\left(-v^{2}+j v / Q\right)
$$

$$
q_{N}=\left(\frac{\alpha}{N}+1-\alpha\right) k_{c}\left(-v^{2}+j v / Q\right),
$$

and

$$
\begin{aligned}
P_{S}(v)= & \frac{2 k_{B} T}{L^{2}} N\left\{\alpha \zeta_{\text {tot }} \sum_{n=1}^{N}\left|H_{n}^{[\text {int }]}\right|^{2}+\left((1-\alpha) N \zeta_{\text {tot }}\right.\right. \\
& \left.\left.+\gamma_{\text {int }}\right)\left|H_{N}^{[\text {int }]}\right|^{2}\right\},
\end{aligned}
$$

where $H_{n}^{[\text {int }]}$ is a function of the complex quantity $\left[k_{\text {int }}+\right.$ $\left.j Q^{-1}\left(k_{c} / \zeta_{\text {tot }}\right) \gamma_{\text {int }} \nu\right]$; see Eq. (A18). We evaluated Eq. (A27) numerically, varying $k_{\text {int }} / k_{c}$ and $\gamma_{\text {int }} / \zeta_{\text {tot }}$, and fitted the obtained $P_{S}(v)$ curves to the SHO model. This resulted in good fits with a low $\chi^{2}$ per data point. From these fits the values for $k_{\text {int }} / k_{c}$ and $\gamma_{\text {int }} / \zeta_{\text {tot }}$ were recalculated and compared with their original values. The systematic errors $\Delta k_{\text {int }}=k_{\text {int }}^{\text {fit }}-k_{\text {int }}$ and $\Delta \gamma_{\text {int }}=\gamma_{\text {int }}^{\text {fit }}-\gamma_{\text {int }}$ have been plotted in Figs. 7 and 8, where $k_{\text {int }}$ has been scaled on $k_{c}$ and $\gamma_{\text {int }}$ on $\zeta_{\text {tot }}$.
[1] L. Bocquet and E. Charlaix, Chem. Soc. Rev. 39, 1073 (2010).

[2] J. C. T. Eijkel and A. van den Berg, Microfluid. Nanofluid. 1, 249 (2005).

[3] B. N. J. Persson and F. Mugele, J. Phys.: Condens. Matter 16, R295 (2004).

[4] M. H. Müser, M. Urbakh, and M. O. Robbins, Adv. Chem. Phys. 126, 187 (2003).

[5] O. Reynolds, Phil. Trans. R. Soc. London 177, 157 (1886).

[6] D. Chan and R. Horn, J. Chem. Phys. 83, 5311 (1985).

[7] F. Mugele and M. Salmeron, Phys. Rev. Lett. 84, 5796 (2000).

[8] J. Israelachvili, Intermolecular and Surface Forces, 2nd ed. (Academic Press, London, 1991).

[9] R. G. Horn and J. N. Israelachvili, Chem. Phys. Lett. 71, 192 (1980).

[10] S. J. O'Shea and M. E. Welland, Langmuir 14, 4186 (1998).

[11] M. Antognozzi, A. D. L. Humphris, and M. J. Miles, Appl. Phys. Lett. 78, 300 (2001).

[12] M. Kageshima, H. Jensenius, M. Dienwiebel, Y. Nakayama, H. Tokumoto, S. P. Jarvis, and T. H. Oosterkamp, Appl. Surf. Sci. 188, 440 (2002).

[13] Y. Zhu and S. Granick, Langmuir 19, 8148 (2003).

[14] T. Becker and F. Mugele, Phys. Rev. Lett. 91, 166104 (2003).

[15] P. D. Ashby and C. M. Lieber, J. Am. Chem. Soc. 126, 16973 (2004).

[16] A. Maali, C. Hurth, T. Cohen-Bouhacina, G. Couturier, and J.-P. Aimé, Appl. Phys. Lett. 88, 163504 (2006).

[17] A. Maali, T. Cohen-Bouhacina, G. Couturier, and J.-P. Aimé, Phys. Rev. Lett. 96, 086105 (2006).

[18] S. Patil, G. Matei, A. Oral, and P. M. Hoffmann, Langmuir 22, 6485 (2006).

[19] G. B. Kaggwa, J. I. Kilpatrick, J. E. Sader, and S. P. Jarvis, Appl. Phys. Lett. 93, 011909 (2008).

[20] L. Bureau and A. Arvengas, Phys. Rev. E 78, 061501 (2008).

[21] W. Hofbauer, R. J. Ho, R. Hairulnizam, N. N. Gosvami, and S. J. O'Shea, Phys. Rev. B 80, 134104 (2009).

[22] L. Bureau, Phys. Rev. Lett. 104, 218302 (2010).
[23] S. H. Khan, G. Matei, S. Patil, and P. M. Hoffmann, Phys. Rev. Lett. 105, 106101 (2010).

[24] S. de Beer, D. van den Ende, and F. Mugele, Nanotechnology 21, 325703 (2010).

[25] S. de Beer, D. van den Ende, and F. Mugele, J. Phys.: Condens. Matter 23, 11206 (2011).

[26] D. Kiracofe and A. Raman, Nanotechnology 22, 485502 (2011).

[27] A. Labuda, K. Kobayashi, K. Suzuki, H. Yamada, and P. Grütter, Phys. Rev. Lett. 110, 066102 (2013).

[28] M. L. Gee, P. M. McGuiggan, J. N. Israelachvili, and A. M. Homola, J. Chem. Phys. 93, 1895 (1990).

[29] J. Klein and E. Kumacheva, Science 269, 816 (1995).

[30] M. Schindler, Chem. Phys. 375, 327 (2010).

[31] A. Labuda and P. Grütter, Langmuir 28, 5319 (2012).

[32] T.-D. Li and E. Riedo, Phys. Rev. Lett. 100, 106102 (2008).

[33] T. E. Schäffer, J. P. Cleveland, F. Ohnesorge, D. A. Walters, and P. K. Hansma, J. Appl. Phys. 80, 3622 (1996).

[34] H. Asakawa and T. Fukuma, Rev. Sci. Instrum. 80, 103703 (2009).

[35] C. Carrasco, P. Ares, P. J. de Pablo, and J. Gómez-Herrero, Rev. Sci. Instrum. 79, 126106 (2008).

[36] S. de Beer, D. van den Ende, and F. Mugele, Appl. Phys. Lett. 93, 253106 (2008).

[37] I. Revenko and R. Proksch, J. Appl. Phys. 87, 526 (2000).

[38] X. Xu, M. Koslowski, and A. Raman, J. Appl. Phys. 111, 054303 (2012).

[39] E. T. Herruzo and R. Garcia, Appl. Phys. Lett. 91, 143113 (2007).

[40] S. J. O'Shea, Phys. Rev. Lett. 97, 179601 (2006).

[41] J. E. Sader and S. P. Jarvis, Phys. Rev. B 74, 195424 (2006).

[42] J. L. Hutter and J. Bechhoefer, Rev. Sci. Instrum. 64, 1868 (1993).

[43] O. H. Willemsen, L. Kuipers, K. O. van der Werf, B. G. de Grooth, and J. Greve, Langmuir 16, 4339 (2000).

[44] J. P. Cleveland, T. E. Schäffer, and P. K. Hansma, Phys. Rev. B 52, R8692 (1995).

[45] F. Benmouna and D. Johannsmann, J. Phys.: Condens. Matter 15, 3003 (2003). 
[46] A. Roters, M. Gelbert, M. Schimmel, J. Rühe, and D. Johannsmann, Phys. Rev. E 56, 3256 (1997).

[47] O. von Sicard, A. M. Gigler, T. Drobek, and R. W. Stark, Langmuir 25, 2924 (2009).

[48] A. Ulcinas, G. Valdre, V. Snitka, M. J. Miles, P. M. Claesson, and M. Antognozzi, Langmuir 27, 10351 (2011).

[49] R. Kubo, Rep. Prog. Phys. 29, 255 (1966).

[50] A. Labuda, M. Lysy, and P. Grütter, Appl. Phys. Lett. 101, 113105 (2012).

[51] N. Wiener, Acta Math. 55, 117 (1930).

[52] A. Khintchine, Math. Ann. 109, 604 (1934).

[53] A. Würger, J. Phys.: Condens. Matter 23, 505103 (2011).

[54] S. de Beer, W. K. den Otter, D. van den Ende, W. J. Briels, and F. Mugele, Europhys. Lett. 97, 46001 (2012).

[55] B. M. Borkent, S. de Beer, F. Mugele, and D. Lohse, Langmuir 26, 260 (2010).

[56] S. F. Nørrelykke and H. Flyvbjerg, Phys. Rev. E 83, 041103 (2011).

[57] J. Klein, Phys. Rev. Lett. 98, 056101 (2007).

[58] C. Cottin-Bizonne, B. Cross, A. Steinberger, and E. Charlaix, Phys. Rev. Lett. 94, 056102 (2005).
[59] See Supplemental Material at http://link.aps.org/supplemental/ 10.1103/PhysRevE.87.062406 for the interaction stiffness meaured by a large tip and the histogram of offsets.

[60] J. E. Sader, J. Appl. Phys. 84, 64 (1998).

[61] A. J. Katan and T. H. Oosterkamp, J. Phys. Chem. C 112, 9769 (2008).

[62] S. J. O’Shea, Jpn. J. Appl. Phys. 40, 4309 (2001).

[63] S. H. Khan and P. M. Hoffmann, arXiv:1210.3540.

[64] P. A Thompson and M. O. Robbins, Phys. Rev. A 41, 6830 (1990).

[65] L.-T. Kong, C. Denniston, and M. H. Müser, Modelling Simul. Mater. Sci. Eng. 18, 034004 (2010).

[66] A. Vadakkepatt, Y. Dong, S. Lichter, and A. Martini, Phys. Rev. E 84, 066311 (2011).

[67] S. de Beer, P. Wennink, M. van der Weide-Grevelink, and F. Mugele, Langmuir 26, 13245 (2010).

[68] B. Q. Luan and M. O. Robbins, Nature 435, 929 (2005).

[69] Y. F. Mo, K. T. Turner, and I. Szlufarska, Nature 457, 1116 (2009).

[70] S. de Beer, W. K. den Otter, D. van den Ende, W. J. Briels, and F. Mugele, Tribol. Lett. 48, 1 (2012). 